\title{
Uso de la retroalimentación correctiva focalizada indirecta con claves metalingüísticas en la adquisición del sufijo -s en la tercera persona del singular en inglés, en estudiantes de un programa de formación pedagógica en EFL de una universidad chilena
}

The Use of Indirect Focused Corrective Feedback with Metalinguistic Clues in the Acquisition of the Suffix -s in the Third Person Singular in

Pre-Service EFL Teachers from a Chilean University

Uso da realimentação corretiva focalizada indireta com senhas metalinguisticas na adquisição do sufixo -s na terceira pessoa do singular em inglês, em estudantes de um programa de formação pedagogica em EfL de uma universidade chilena

Mabel Andrea Ortiz ${ }^{1}$

Resumen

El presente artículo aborda uno de los problemas recurrentes en estudiantes de un programa de formación de profesores de inglés en Chile: la omisión de la -s en la tercera persona del singular durante la construcción de un texto académico. Se ha observado que las competencias gramaticales de estos estudiantes son insuficientes para responder a las exigencias de la producción de un texto académico. Esta problemática deja en evidencia una situación común entre los estudiantes: avanzar en su competencia comunicativa, pero no en su competencia gramatical. El futuro profesor de inglés como lengua extranjera (EFL) debe poseer un dominio en ambas competencias y, por lo tanto, se debe brindar atención a ambos aspectos. El artículo describe también la utilidad de las estrategias de retroalimentación correctiva, específicamente de la retroalimentación correctiva focalizada indirecta con claves metalingüísticas para abordar problemas gramaticales concretos y recurrentes, como el de la omisión del sufijo -s, en estudiantes con un nivel de competencia comunicativa avanzada en inglés.

Palabras clave: Sufijo -s, retroalimentación correctiva, escritura académica

Abstract

This article addresses one of the frequent problems in pre-service teachers of English in Chile: the omission of suffix -s in the third person singular during the writing of an academic text. It has been observed that the grammatical competence of these prospective teachers is insufficient to meet the demands of academic writing. This issue reveals a situation that is shared by students who show proficiency in their communicative competence but not in their grammatical competence. The prospective teacher of English as a Foreign Language should master both competences. Therefore, attention should be equally given to both. The paper also describes the usefulness of corrective feedback strategies, specifically indirect focused corrective feedback with metalinguistic clues to address specific, frequent grammatical problems, such as the omission of suffix -s in students who show advanced proficiency in their communicative competence.

Keywords: Suffix -s, corrective feedback, academic writing

1 Académica del Departamento de Lenguas de la Facultad de Educación. Universidad Católica de la Santísima Concepción, Chile.Correo electrónico: mortiz@ucsc.cl. 
Este artigo aborda um dos problemas recorrentes em alunos de um programa de formação para professores de Inglês no Chile: a omissão de -s na terceira pessoa do singular durante a construção de um texto acadêmico. Tem-se observado que as habilidades gramaticais destes estudantes são insuficientes para atender as demandas da produção de um texto acadêmico. Este problema revela uma situação comum entre os estudantes: avançar a sua competência comunicativa, mas não em sua competência gramatical. 0 futuro professor de Inglês como língua estrangeira (EFL) deve ter um domínio em ambas competições e, portanto, deve ser dada atenção para dois aspectos. 0 artigo também descreve a utilidade de estratégias de retroalimentação correctivas, especificamente da retroalimentação focalizada corretiva indireta com chaves metalinguísticas para resolver os problemas gramaticais específicos e recorrentes, como a omissão do sufixo -s em alunos com um nível básico de competência comunicativa em inglês.

Palabras chave: Sufixo-s, retroalimentação corretiva, escritura acadêmica

\section{Artículo recibido el 11 de agosto de 2015 y aprobado el 27 de enero de 2016}

\section{Introducción}

Todo estudiante, durante el proceso de adquisición de una segunda lengua (L2), debe enfrentarse con frecuencia a distintos tipos de obstáculos. Dentro de ellos podemos encontrar los errores lingüísticos que se cometen en su producción oral y escrita. En este contexto, se considera un aspecto problemático el tema de la precisión lingüística, en otras palabras, la habilidad para producir enunciados gramatical, léxica y fonológicamente correctos (Housen \& Kiuken, 2009). La precisión lingüística es uno de los principales focos de atención de los profesores de inglés en Chile. Al respecto, algunas investigaciones señalan que, en situaciones en las cuales el idioma inglés no se encuentra en un contexto inmediato, como es el caso de nuestro país, los docentes consideran los errores gramaticales como distractores y estigmatizadores, puesto que pueden interferir en la comprensión del mensaje (Ferris \& Robert, 2001, p. 14). Otros estudios llevados a cabo por Hedgock y Lefkowitz (1996) indican que los estudiantes de inglés como lengua extranjera, en comparación con los estudiantes de inglés como L2, manifiestan un interés en que les corrijan aspectos específicos de la lengua, tales como, gramática o léxico. Los errores gramaticales más frecuentes encontrados en los textos escritos de estudiantes de inglés como lengua extranjera corresponden a construcciones pasivas, uso de verbos modales y tiempos verbales.

El presente artículo se centra en uno de los errores recurrentes en la escritura, que no puede ser ignorado: la omisión de la -s en la tercera persona del singular en la escritura académica en inglés.

Segundo semestre de 2016 • pp 127-136
La necesidad de focalizarse específicamente en este tipo de error surge, por una parte, a partir de la experiencia académica como docente de inglés y formadora de profesores de inglés; $y$, por otra, gracias a las investigaciones pertinentes al tema, encontradas durante la revisión de la literatura y que abordan la importancia de focalizar el análisis en ciertos tipos de errores gramaticales. Con relación a la utilización de estrategias de retroalimentación correctiva, Hyland \& Hyland (2006), Ellis (1997), Sheen (2011), entre otros, señalan que las investigaciones en la adquisición de una L2 indican que el feedback dirigido solamente a un rasgo lingüístico tendría un efecto positivo en la adquisición de dicho rasgo.

\section{La s final en la tercera persona del singular}

Las investigaciones en el campo de adquisición de segundas lenguas concuerdan en la dificultad que implica adquirir algunos morfemas gramaticales. Tal es el caso de la $s$ final, que es una de las flexiones del verbo en inglés y se agrega al final de este en tercera persona singular. Si bien este morfema ha sido considerado una forma básica para los aprendices de inglés como L2 (Brown, 2000; Hsieh, 2009; O'Grady, 2006), se ha llegado a la conclusión de que los estudiantes experimentan una particular dificultad para adquirir este morfema, independientemente del nivel de competencia comunicativa que hayan logrado. En otras palabras, se ha observado que, sin importar el nivel de adquisición del inglés como L2, es recurrente que los estudiantes omitan la $s$ final del verbo en construcciones de tercera persona. 
Uso de la retroalimentación correctiva focalizada indirecta con claves metalingüísticas en la adquisición del sufijo -s en la tercera persona del singular en inglés Mabel Andrea Ortiz

De acuerdo con Ellis (1997), la expresión morfemática del pasado regular y la de la tercera persona singular se consideran unas de las más difíciles de adquirir en inglés como L2. Esto comprueba que no existe una relación directa entre la complejidad lingüística y el aprendizaje (Lightbown \& Spada, 2008). Es decir, si bien algunas estructuras pueden ser lingüísticamente fáciles, como la -s en la tercera persona, no son fáciles de aprender o internalizar para hablantes no nativos. Esto podría explicar el porqué algunos estudiantes avanzan en su competencia comunicativa, pero no así en su competencia gramatical. Algunas investigaciones en este ámbito han demostrado que existen etapas de desarrollo en la adquisición de la morfología y la sintaxis de una L2 (Johnson \& Johnson, 2014; Lightbown \& Spada, 2000); sin embargo, no hay acuerdos sobre este supuesto orden. Con relación a la $s$ final, algunos estudios (Goldschneider \& Dekeyser, 2001; Hsieh, 2009; Shin \& Milroy, 1999) han arrojado como resultado que la -s se encuentra en uno de los niveles más difíciles de adquirir. $\mathrm{Al}$ respecto, autores como Pienemann (2005) y Ellis (2006) intentan explicar la dificultad que implica la adquisición de la -s final, a través de dos teorías diferentes.

Pienemann (2005), en su 'teoría de la procesabilidad', señala que el estudiante de una L2 adquiere solo aquellas estructuras lingüísticas que es capaz de procesar. De acuerdo a esta teoría, los alumnos adquirirían las estructuras siguiendo un orden estricto, que es independiente de la L1; por lo tanto, para avanzar de un nivel a otro en la adquisición de una L2, se necesita haber aprendido el nivel anterior. Esto explicaría por qué los estudiantes no pueden adquirir todas las estructuras gramaticales de una sola vez. Brown \& Larson- Hall (2012) plantean que si bien cada individuo adquiere las estructuras de manera diferente, los estudiantes que comparten la misma L1 deberían seguir un mismo orden de adquisición. En el contexto de la adquisición de la $s$ final, Pienemann (2005) señala que este morfema es la última estructura dentro del orden jerárquico y, por lo tanto, los alumnos no la dominarán mientras no manejen todos los elementos que están en el nivel anterior.
Por su parte Ellis (2009), en su 'teoría asociativa del aprendizaje', destaca dos palabras clave dentro del proceso de adquisición de ciertos morfemas: 'bloqueo' y 'desinterés'. De acuerdo con este autor, el proceso de bloqueo se produce cuando se presentan dos o más elementos lingüísticos que componen un significado. Así, los estudiantes adquieren el elemento más destacado, lo que lleva a desatender el aprendizaje de los menos relevantes. En el caso del presente estudio, el sufijo -s sería el menos relevante, mientras que el más destacado sería el aprendizaje de las partes constitutivas de la oración. Al respecto, el autor sostiene que "el bloqueo puede ser mayor cuando no existe un equivalente de la L2 en la L1. Una vez que el estudiante omite regularmente una estructura lingüística es difícil revertir este proceso" (2009, p. 68). Esta aseveración del autor reafirma las dificultades lingüísticas por las que atraviesa un estudiante que adquiere una segunda lengua.

Otra explicación acerca de la omisión o uso incorrecto de la $s$ final hace referencia a las disparidades entre L1 y L2. De acuerdo con Odlin "las similitudes entre la L1 y la L2 ayudan a los alumnos en la adquisición y, por el contrario, las diferencias impiden su adquisición" (2003, p. 54). De este modo, se infiere que la omisión o el uso incorrecto de la $s$ final tienen relación con la inexistencia de este morfema en la L1. Otros estudios (Brown, 2007; Gass \& Selinker, 2009; Selinker, 1992) señalan que no todos los errores tienen su origen en la interferencia de la L1. Así por ejemplo, el uso del morfema -ed en los verbos irregulares en el pasado simple constituye un error común tanto en los hablantes de inglés como L1 como en quienes lo aprenden como L2. Por lo tanto, los errores que cometen los estudiantes no siempre derivan de la L1, sino que se atribuyen también al proceso de interlengua, conocido también como el sistema lingüístico de transición que resulta de la combinación de la L1 con la L2, mientras se aprende esta última (Baralo, 2004; Yang, 2010). Hay que precisar que el nuevo sistema lingüístico corresponde al de un estudiante no nativo y consiste en una serie de etapas que experimenta el aprendiz hasta llegar o aproximarse al nivel de competencia deseado en la L2 (véase la figura 1). 
Universidad Pedagógica Nacional

Facultad de Humanidades

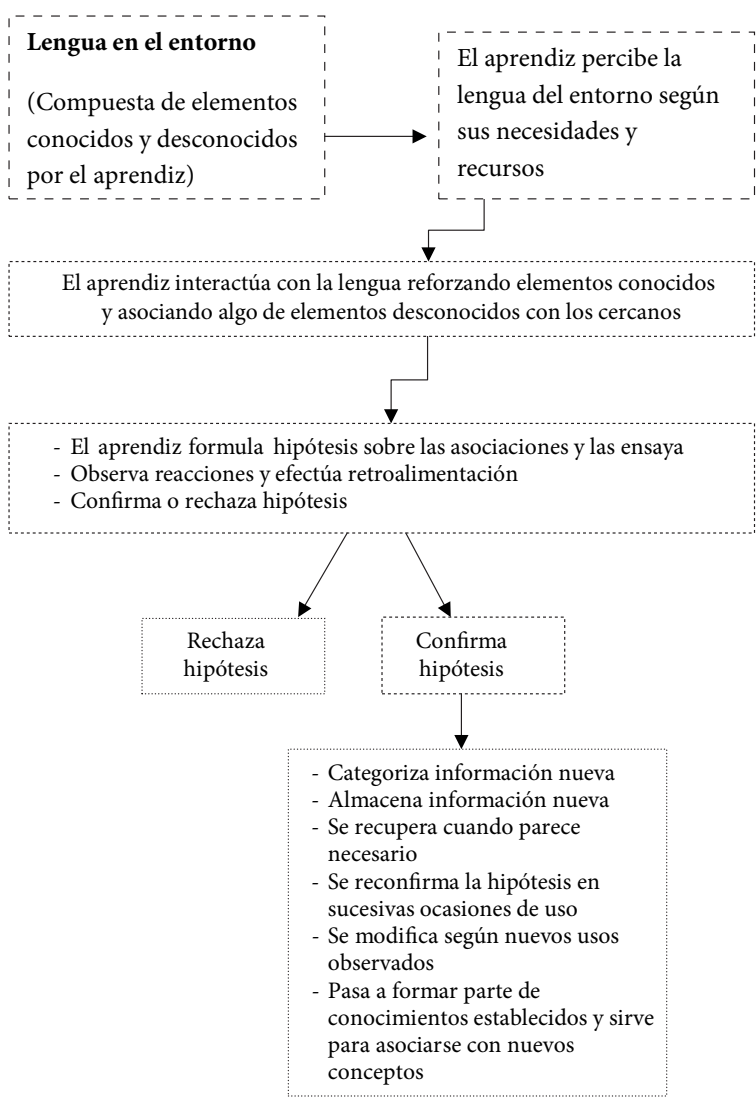

Figura 1. Proceso de interlengua. Fuente: Tomado de Griffin (2005, p. 97).

Tal como se muestra en la figura 1, durante el proceso de adquisición de la L2 el estudiante construye sus propias hipótesis sobre la estructura de la lengua. En este sentido, Yule (1996) plantea que "los errores pueden ser causados por la influencia de la primera lengua; otra, por la generalización excesiva de reglas adquiridas, o simplemente son propios del proceso de aprendizaje o del proceso creativo y cognitivo del aprendiz" (p. 195). Así, mientras el estudiante evoluciona en su aprendizaje y apropiación de la L2, llega un momento en que él crea un sistema propio de reglas, que no corresponde ni a la primera ni a la segunda. Por lo tanto, si bien se trata de un proceso intermedio, es un sistema que posee un carácter individual. En este proceso el estudiante utiliza e interpreta de distintas maneras la información que posee sobre la L2, por ejemplo, interpretar una lengua a partir de la traducción literal, esto es, traducir la L2 siguiendo la estructura de la L1. Atendiendo al tema en estudio, si el estudiante lleva a cabo una interpretación literal, lo más probable es que, en una tarea de escritura, tienda a omitir el sufijo -s en la tercera persona del singular en inglés.

En consecuencia, las características de la interlengua evidencian que el aprendizaje de una segunda lengua es un proceso complejo, aún más cuando se produce la fosilización. Este fenómeno lingüístico, que consiste en la retención en la interlengua de manera inconsciente, de algunos elementos propios de la L1 (Arcos, 2009; Baralo, 2004), tiene dentro de sus posibles causas la ausencia de retroalimentación correctiva, según plantea Bustos (2006). La fosilización puede producirse en cualquier nivel de aprendizaje y es muy común en el proceso de adquisición de una L2. De ahí la importancia que puede tener una estrategia de reparación o la provisión de retroalimentación correctiva para el tratamiento de los errores gramaticales. Al no existir tal corrección, el estudiante no detecta el error y lo repite. Ante la presencia de este fenómeno, es evidente que el estudiante necesita ser corregido, independientemente de que su fluidez y su competencia comunicativa no representen un problema.

Ahora bien, mucho se ha teorizado acerca de cómo debe abordarse un error gramatical durante el proceso de enseñanza y aprendizaje de una L2. Específicamente, cuál debe ser la manera de actuar del docente frente a un error de los estudiantes. Lo anterior supone un gran reto para el profesor, ya que las decisiones sobre "el cómo y cuándo se deberían corregir los errores y quién debería hacerlo" (Ellis, 1997, p. 64) puede repercutir en aspectos significativos de la enseñanza y aprendizaje de la L2.

\section{El rol de las estrategias de retroalimentación correctiva}

El rol de la retroalimentación correctiva en la adquisición de lenguas ha sido siempre un tema controvertido (Tatawy, 2002). Algunas teorías otorgan importancia a estas estrategias en la adquisición de una segunda lengua, mientras que otras no distinguen su utilidad. Teorías como la gramática universal de Chomsky (2004) y la hipótesis del input de Krashen (1982), por ejemplo, no le conceden un 
Uso de la retroalimentación correctiva focalizada indirecta con claves

metalingüísticas en la adquisición del sufijo -s en la tercera persona del singular en inglés

Mabel Andrea Ortiz

rol relevante a las estrategias de retroalimentación correctiva.

De acuerdo a la teoría de la gramática universal, cuyo precursor es Chomsky, la retroalimentación correctiva no tendría ninguna utilidad. De esta manera, el ser humano nacería con una capacidad innata para aprender un idioma y, por lo tanto, no dependería de ningún estímulo para adquirirla (Chomsky, 2004; Gass et ál., 2013). Por su parte, la teoría del input comprensible de Krashen (1982), sostiene que "para que haya adquisición solo es necesaria la exposición a un input natural no secuenciado gramaticalmente y la participación en la interacción sin necesidad de ningún feedback negativo, ya que los mecanismos internos se encargan del proceso" (p. 6). En otras palabras, tanto el input como la interacción serían elementos suficientes para la adquisición de una L2. Lo anterior, especialmente, cuando se trata de la adquisición de ciertas formas.

Por otra parte, entre las teorías que reconocen en la retroalimentación correctiva un rol importante destacan las teorías interaccionistas, tales como la hipótesis de la interacción de Long (1996), la hipótesis del output de Swain (1995), la hipótesis del noticing de Schmidt (1994), y la teoría sociocultural de Vygotsky (1999). Las teorías interaccionistas promueven la eficacia de la retroalimentación correctiva durante el proceso de interacción comunicativa. Así, en el momento de producir un mensaje, los estudiantes prestan atención, principalmente, al significado. Por esta razón, deben recibir una retroalimentación correctiva cuando cometen un error, ya sea de parte del docente o de un par. En este contexto, Ellis (2009) plantea que "la retroalimentación correctiva facilita la adquisición mediante la activación de los procesos internos, tales como la atención y la repetición, que hacen posible la adquisición” (p. 12). Tales retroalimentaciones pueden darse en el contexto de un diálogo entre profesor y alumno, a través de la clarificación, de la repetición o de algún otro tipo de señal frente al error cometido.

De acuerdo a la teoría sociocultural, la retroalimentación correctiva también cumple un papel esencial. Sin embargo, difiere de las teorías interaccionistas, al sostener que la adquisición de una L2 no es un proceso individual. Tal como plantea Ellis (2009), para la teoría sociocultural la adquisición ocurre durante la interacción y no después de esta, es decir, necesariamente involucra a otro individuo. Asimismo, Anton (1999), citado en Ellis, señala que "la interacción dialógica permite a un experto (como un profesor) crear un contexto en el cual un novato puede participar activamente de su propio aprendizaje y en el que el experto puede apoyar este proceso" (2009, p. 22). Así, en este proceso dialógico, la retroalimentación correctiva se concibe como un componente que fomenta el aprendizaje autorregulado, aspecto que promueve la teoría sociocultural. En otras palabras, la retroalimentación correctiva, proporcionada por alguien con mayores habilidades y conocimientos, facilitaría y ayudaría al que aprende en su proceso de reconocimiento y corrección de errores de manera independiente.

La provisión de retroalimentación correctiva conlleva entregar un tipo de información que los estudiantes puedan utilizar para la modificación de sus errores. Los estudiantes que no tienen la posibilidad de la práctica del inglés en su contexto inmediato, como sucede con los estudiantes chilenos, necesitan de mayores instancias de retroalimentación por parte de sus profesores. En este contexto, la retroalimentación correctiva sí se considera un elemento primordial, especialmente en aquellos estudiantes que adquieren un segundo idioma con fines pedagógicos y que al mismo tiempo se encuentran en una etapa en que logran una comunicación efectiva, pero requieren de una mayor precisión lingüística. Esta estrategia puede ayudar al estudiante, por una parte, a desarrollar mayor claridad sobre su propio proceso de adquisición, y por otra, a transitar más rápidamente por los diferentes estados de aprendizaje de la lengua extranjera, entre ellos la etapa de interlengua. En esta etapa, la retroalimentación puede servir al estudiante para confirmar o rechazar sus hipótesis relacionadas con aspectos gramaticales y contribuir de esta manera a prevenir la fosilización lingüística. 
Universidad Pedagógica Nacional

Facultad de Humanidades

Las investigaciones sobre el papel de la retroalimentación correctiva se han centrado principalmente en el discurso oral. Sin embargo, la importancia de entregar retroalimentación correctiva en el discurso escrito ha recibido mayor atención en los últimos años. En este contexto, teóricos como Kepner (1991), Semke (1984) y Sheppard (1992), entre otros, influenciados por los enfoques de la escritura como proceso, señalan que la corrección de errores tiene poca incidencia o efecto en la producción escrita de los estudiantes. Asimismo, Truscott (1996), uno de los principales detractores de la retroalimentación correctiva, afirma que la corrección gramatical no tiene lugar en los cursos de producción escrita y, por ende, no debería ser considerada. El teórico apoya su idea en la falta de evidencia respecto al hecho de que la corrección de errores gramaticales ayude realmente a los estudiantes a mejorar la precisión lingüística de sus escritos. Ferris (2011), por su parte, plantea que las opiniones de Truscott (1996) son muy prematuras y enfatiza que la corrección de errores puede y ayuda, en gran medida, a algunos estudiantes en el avance de la precisión lingüística en la producción escrita. Las opiniones de Ellis pueden ser corroboradas por algunos estudios empíricos (Bitchener, 2008; Bitchener \& Knoch, 2008, 2009; Dekeyser, 2005; Ferris, 2010; Ferris \& Roberts, 2001; Lee, 1997 \& Sheen, 2007, 2010), que si bien no son concluyentes, han demostrado que retroalimentación correctiva en la corrección de errores sí puede aportar en la adquisición de una L2. Dichos estudios dan cuenta del efecto de ciertos tipos de retroalimentación correctiva en la adquisición de una L2, con una atención marcada en la forma.

\section{Las estrategias de retroalimentación focalizada}

Algunos autores (Ferris \& Roberts, 2001; Han, 2002; Sheen, 2007, 2010, entre otros) que destacan la importancia de la provisión de retroalimentación correctiva, señalan que la retroalimentación correctiva focalizada o proporcionada a una o dos estructuras lingüísticas puede contribuir de manera más satisfactoria a la adquisición de dichas estructuras. En este contexto, surge la pregunta $i$ Es necesario corregir todos los errores que comenten los estudiantes en un texto? Al respecto, Cassany plantea que

[...] muchas veces el alumno no puede asimilar de una vez un número muy elevado de correcciones, puesto que en alumnos principiantes habrá errores demasiado difíciles para su nivel, otros que se tratarán en lecciones venideras, y algunos de poca importancia. (2011, p. 41)

En el contexto nacional, se ha observado que los docentes de inglés en Chile generalmente tienden a corregir todos los tipos de errores en los textos escritos por sus estudiantes. Al respecto, es poco probable que los estudiantes puedan darse cuenta de sus errores gramaticales si tienen que prestar atención a más de dos o tres categorías de errores, dado que la adquisición de un idioma es un proceso lento y complejo. Por lo tanto, atender a un gran número de errores puede sobrecargar la capacidad cognitiva de los estudiantes, como lo señalan Cots, Armengol, Arnó, Irún \& Llurda (2007), Cassany (2011) y Hyland (2003). No todos los estudiantes pueden asimilar un gran número de errores, más aún cuando los errores son de índole gramatical, que es cuando los estudiantes evidencian mayores debilidades lingüísticas. Desde esta perspectiva, se deduce que cuanto menos agobiante sea el proceso de corrección de errores para el estudiante, mayor será su capacidad para atender a los errores en sus textos escritos.

Del mismo modo, focalizar la atención en algunos errores puede tener una incidencia positiva en la motivación del estudiante. Si bien la motivación es esencial en todas las disciplinas, en el aprendizaje de un idioma es significativamente relevante dado que es un proceso complejo y algunas de las causas importantes por las que estos interrumpen el aprendizaje de una lengua serían la frustración y la desmotivación. Por lo tanto, el factor motivacional en el aprendizaje de una lengua no puede ser ignorado y como lo han corroborado algunos autores (Cots, Armengol, Arnó, Irún \& Llurda, 2007; Moure \& Palacios, 1996) existe una relación positiva 
Uso de la retroalimentación correctiva focalizada indirecta con claves

metalingüísticas en la adquisición del sufijo -s en la tercera persona del singular en inglés

Mabel Andrea Ortiz

entre motivación y el éxito en el aprendizaje de una lengua, puesto que promovería en el estudiante una participación más activa. Desarrollar una actitud más activa se condice con la disposición que se espera en el aula hoy en día y, por lo tanto, constituye un factor que puede incidir de manera decisiva en los procesos de adquisición de una L2. En el contexto de este estudio, se puede inferir entonces que al estar el estudiante más motivado, mayor será su atención a la retroalimentación de su profesor. De esta manera, es esencial la búsqueda de estrategias que fomenten una actitud positiva del estudiante, si se desea lograr mejores aprendizajes.

En otro orden de ideas, las estrategias de retroalimentación deben promover la autonomía del futuro profesor de inglés. Desde esta perspectiva, un tipo de retroalimentación correctiva focalizado que puede promover esta autonomía es la retroalimentación correctiva indirecta metalingüística.

\section{Retroalimentación correctiva indirecta metalingüística}

Una retroalimentación indirecta es aquella en que el docente indica al estudiante que ha cometido un error, pero sin entregar la forma correcta. Algunos estudios plantean que la retroalimentación indirecta puede tener, con el transcurso del tiempo, iguales o mejores efectos en la precisión lingüística que una retroalimentación directa (Ferris \& Helt, 2000; Frantzen, 1995). Del mismo modo, algunos autores señalan que la retroalimentación indirecta cambiaría la percepción de los estudiantes sobre la responsabilidad en su propio aprendizaje (Chandler, 2003; Srichanyachon, 2012). En este contexto, la retroalimentación indirecta promovería el pensamiento crítico en el alumno y contribuiría a que su rol fuera mucho más activo.

Un tipo de retroalimentación correctiva focalizada, que promueven autores como Bitchener (2008), Bitchener \& Knoch (2009) y Sheen (2011), es aquel que entrega claves metalingüísticas. En concreto, el componente metalingüístico implica la capacidad del estudiante de reflexionar sobre el lenguaje e ir más allá del uso de la lengua. El uso de claves metalingüísticas implica que la atención se dirige al lenguaje mismo y conlleva una reflexión más profunda y consciente sobre lo que se está aprendiendo. De este modo, la reflexión metalingüística puede contribuir a un aprendizaje más eficaz de la lengua según diversos autores (Cots, Armengol, Arnó, Irún \& Llurda, 2007; Bitchener, 2008; Bitchener \& Knoch, 2008; Sheen, 2011). Ahora bien, el tipo de retroalimentación que entregue el profesor estará determinado por el nivel de competencia del estudiante, puesto que para un estudiante no nativo que posee un nivel de conocimiento básico de la L2 resultaría incomprensible la información metalingüística. Esto implica que el estudiante debe estar en una etapa del proceso de producción que le permita llegar a esta conciencia metalingüística. Por lo tanto, la utilización de una retroalimentación indirecta metalingüística va dirigida a un futuro profesor de inglés con un conocimiento avanzado de la L2.

La provisión de una retroalimentación correctiva indirecta con claves metalingüísticas puede promover no solo la autonomía en el futuro profesor de inglés, sino animarlos a hacer uso de la lengua para hablar de ella misma y, tal vez más importante aún, promover la autocorrección. Esta última puede ayudar significativamente a la autonomía del estudiante y crear conciencia en él sobre su propia manera de aprender y adquirir la estructura lingüística de la lengua meta. Su desarrollo forma parte de la formación del docente y es por esto que una de las responsabilidades del formador de profesores es diseñar actividades que favorezcan los procesos cognitivos, que promuevan la autocorrección, el autocontrol o la autorregulación de las actividades metacognitivas del futuro profesor de inglés y la reflexión en torno al aprendizaje. Al respecto, el docente de lenguas no siempre estimula el desarrollo de estos procesos en el aula, ya sea por desconocimiento o porque se centra principalmente en la entrega de contenidos o de conocimientos elaborados y no en el desarrollo de estrategias de aprendizaje. 
Universidad Pedagógica Nacional

Facultad de Humanidades

\section{Algunas conclusiones}

A partir de lo señalado se pueden presentar varias conclusiones. Primero, la utilización de una retroalimentación correctiva focalizada es una alternativa que los docentes deberían considerar en la producción escrita. Esto no significa atender solamente a algunos errores y dejar otros de lado, sino centrarse en un tipo de error en un texto y después abarcar otros en otra instancia, más aún cuando los errores son sistemáticos.

Segundo, una retroalimentación correctiva proporcionada de manera indirecta y apoyada por claves metalingüísticas puede ser relevante en la corrección de errores, dado que propicia los procesos de reflexión y la búsqueda de respuestas por parte del estudiante frente a dudas sobre la lengua que se estudia. Todo buen profesor debería promover la reflexión entre los futuros profesores a través de este tipo de retroalimentación, no solo porque esta estrategia puede ayudar al estudiante en su proceso de adquisición de una estructura gramatical, sino que también lo involucra en la corrección de sus errores.

La no provisión de claves metalingüísticas puede ser una de las causas importantes que explican el porqué los estudiantes siguen cometiendo los mismos tipos de errores en sus textos. $\mathrm{Al}$ respecto, no es común que los estudiantes reciban explicaciones metalingüísticas sobre sus errores y, en el caso de que se hagan, estas no son frecuentes ni sistemáticas. Por lo tanto, se puede deducir que la utilización de claves metalingüísticas en un estudiante que adquiere el idioma como parte de su formación de profesor de inglés es aún más relevante, puesto que el futuro docente no solo debe fortalecer el conocimiento pragmático de la lengua, sino también el conocimiento declarativo. En otras palabras, la competencia en el uso de la gramática de un futuro docente de inglés debe ser superior a la de un estudiante que adquiere una L2 con fines comunicativos. Esto nos recuerda además la importancia del uso y manejo del metalenguaje en el profesor de lenguas y en el desarrollo de este durante su formación académica.
Finalmente, los estudiantes difícilmente adquirirán estructuras como el morfema -s de manera inconsciente porque aprenden una lengua sintácticamente distinta a la L1, en la que no hay un equivalente, como sucede entre el idioma español y el idioma inglés. Se debe inducir al estudiante a darse cuenta y a poner atención a sus errores, dado que este paso es considerado el primero para lograr la adquisición (Gass, 1988; VanPatten, 2011; Zhang, 2012). Más aún, si el contexto no permite un uso inmediato del idioma inglés fuera del aula, se hace imprescindible el desarrollo de un mayor grado de conciencia sobre las estructuras lingüísticas más difíciles de adquirir para los estudiantes.

En conclusión, el uso frecuente de retroalimentación correctiva, especialmente una retroalimentación focalizada indirecta con claves metalingüísticas, podría ayudar a prevenir muchos de los problemas de fosilización lingüística, más aún en estudiantes que requieren de un trabajo largo y arduo para lograr el dominio de una L2. Tal es el caso de los estudiantes en Chile.

\section{Referencias bibliográficas}

Anton, M. (1999). The Discourse of a learner-centered classroom: Sociocultural perspectives on teacherlearner interaction in the second language classroom. The Modern Language Journal, 83 (3), 303-318.

Arcos, M. (2009). Análisis de errores, contrastivo e interlengua, en estudiantes brasileños de español como L2: verbos que rigen preposición y/o ausencia de ella (tesis doctoral). Universidad Complutense de Madrid. Madrid.

Baralo, M. (2004). La interlengua del hablante no nativo. En J. Sánchez Lobato, I. Santos Gargallo y R. Pinilla (eds.) Vademécum: enseñanza y aprendizaje del español como segunda lengua y como lengua extranjera (pp. 369-389). Madrid: SGEL.

Bitchener, J. (2008). Evidence in support of written corrective feedback. Journal of Second Language Writing, 17 (2), 102-118.

Bitchener, J. \& Knoch, U. (2008). The value of written corrective feedback for migrant and international students. Language Teaching Research, 12 (3), 409-431. 
Bitchener, J., \& Knoch, U. (2009). The relative effectiveness of different types of direct written corrective feedback. System, 37 (2), 322-329.

Brown, H. (2000). Principles of language learning and teaching, 4th ed. Nueva York: Addison Wesley Longman.

Brown, H. D. (2007). Principles of language learning and teaching. Nueva York: Pearson Education.

Brown, S. \& Larson-Hall, J. (2012). Second language acquisition myths: Applying second language research to classroom teaching. Ann Arbor, MI: University of Michigan Press.

Bustos J. M. (2006). Principios metodológicos para el estudio de la fosilización en el aprendizaje del español como lengua extranjera: el buen aprendiz. En J. M. Bustos \& J. Sánchez Iglesias (coord.). La estabilización en el aprendizaje/adquisición del español como L2: el buen aprendiz. Salamanca: Hispano Lusa de Ediciones.

Cassany, D. (2011). La cocina de la escritura. Barcelona: Edición Anagrama.

Chandler, J. (2003). The efficacy of various kinds of error feedback for improvement in the accuracy and fluency of L2 student writing. Journal of Second Language Writing, 12 (3), 267-296.

Chomsky, N. (2004 [1974]). Estructuras sintácticas (14. ${ }^{a}$ ed. en español). México: Siglo Veintiuno.

Cots, J.; Armengol, L.; Arnó, E.; Irún, M. \& Llurda, E. (2007). La conciencia lingüística en la enseñanza de lenguas. Barcelona: Graó.

DeKeyser, R. (2005). What makes learning secondlanguage grammar difficult? A Review of issues. Language Learning, 55 (S1), 1-25.

Ellis, R. (1997). Second language acquisition. In Oxford introduction to linguistics. Oxford: Oxford University Press.

Ellis, R. (2006). Researching the effects of form-focused instruction on L2 acquisition. AILA Review, 19 (1), $18-41$.

Ellis, R. (2009). A typology of written corrective feedback types. ELT Journal, 63 (2), 97-107.

Ferris, D. (2010). Second language writing research and written corrective feedback in SLA. Studies in Second Language Acquisition, 32 (2), 181-201.

Ferris, D. (2011). Treatment of error in second language student writing. Ann Arbor, MI: University of Michigan Press.
Uso de la retroalimentación correctiva focalizada indirecta con claves metalingüísticas en la adquisición del sufijo -s en la tercera persona del singular en inglés Mabel Andrea Ortiz

Ferris, D. \& Roberts, B. (2001). Error feedback in L2 writing classes: How explicit does it need to be? Journal of second language writing, 10 (3), 161-184.

Ferris, D. \& Helt, M. (2000). Was Truscott right? New evidence on the effects of error correction in L2 writing classes. Ponencia presentada en el Congreso de la American Association of Applied Linguistics, marzo 11-14. Vancouver, BC.

Frantzen, D. (1995). The effects of grammar supplementation on written accuracy in an intermediate Spanish content course. The Modern Language Journal, 79 (3), 329-344.

Gass, S. (1988). Integrating research areas: a framework for second language studies. Applied Linguistics 9, 198-217.

Gass, S. \& Selinker, L. (2009). Second language acquisition. Nueva York: Routledge.

Gass, S.; Behney, J. \& Plonsky, L. (2013). Second language acquisition. An introductory course ( $4^{\text {th }}$. ed.). Nueva York: Routledge.

Goldschneider, J. \& DeKeyser, R. (2001). Explaining the "natural order of L2 morpheme acquisition" in English: A meta-analysis of multiple determinants. Language Learning, 51 (1), 1-50.

Griffin, K. (2005). Lingüística aplicada a la enseñanza del español como 2/L. Manuales de formación de profesores de español 2/L. Madrid: Arco Libros.

Han, Z. (2002). A study of the impact of recasts on tense consistency in L2 output. Tesol Quarterly, 36 (4), 543-572.

Hedgcock, J. \& Lefkowitz, N. (1996). Some input on input: Two analyses of student response to expert feedback in L2 writing. The Modern Language Journal, 80 (3), 287-308

Hsieh, F. (2009). The acquisition of English tense/agreement morphology and copula "be" by L1-Chinesespeaking learners. Disponible en http://www.ling. lancs.ac.uk/pgconference/v03/Hsieh.pdf

Housen, A. \& Kuiken, F. (2009). Complexity, accuracy, and fluency in second language acquisition. Applied Linguistics, 30 (4), 461-473.

Hyland, F. (2003). Focusing on form: student engagement with teacher feedback. System, 31 (2), 217-230.

Hyland, K. \& Hyland, F. (2006). Feedback in second language writing: Contexts and issues. Cambridge: Cambridge University Press. 
Universidad Pedagógica Nacional

Facultad de Humanidades

Johnson, D. \& Johnson, F. (2014). Joining together: Group theory and group skills. Boston, MA: Allyn \& Bacon Cambridge: Cambridge University Press.

Kepner, C. (1991). An experiment in the relationship of types of written feedback to the development of second-language writing skills. The Modern Language Journal, 75 (3), 305-313.

Krashen, S. (1982). Principles and practice in second language acquisition. Oxford: Pergamon.

Lee, I. (1997). ESL learners' performance in error correction in writing: Some implications for teaching. System, 25 (4), 465-477.

Lightbown, P. \& Spada, N. (2000). How languages are learned. Oxford: Oxford University Press.

Lightbown, P. \& Spada, N. (2008). How languages are learned. United Kingdom. Oxford University Press, 42 (2), 181-207.

Long, M. (1996). The role of the linguistic environment in second language acquisition. Handbook of Second Language Acquisition, 2, 413-468.

Moure, T. \& Palacios, I. (1996). La didáctica de las lenguas extranjeras: lingüística aplicada en el ámbito académico. En M. Fernández Pérez (coord.). Avances en lingüística aplicada, 47-103.

Odlin, T. (2003). Language transfer. Cambridge. Cambridge: University Press.

O'Grady, W. (2006). The problem of verbal inflection in second language acquisition. Disponible en http:// www. ling.hawaii.edu/faculty/ogrady/Verbal_inflection_in_SLA.pdf.

Pienemann, M. (2005). Cross-linguistic aspects of processability theory. Amsterdam: John Benjamins.

Selinker, L. (1992). Rediscovering language. Essex: Longman.

Semke, H. (1984). Effects of the red pen. Foreign Language Annals, 17 (3), 195-202.

Sheen, Y. (2007). The effect of focused written corrective feedback and language aptitude on EsL learners' acquisition of articles. Tesol Quarterly, 41 (2), 255-283.

Sheen, Y. (2010). Differential effects of oral and written corrective feedback in the ESL classroom. Studies in Second Language Acquisition, 32 (2), 201-234.
Sheen, Y. (2011). Corrective feedback, individual differences and second language learning. Dordrecht: Springer.

Sheppard, K. (1992). Two feedback types: Do they make a difference. RELC Journal, 23 (1), 103-110.

Shin, S. \& Milroy, L. (1999). Bilingual language acquisition by Korean schoolchildren in New York City. Bilingualism: Language and Cognition, 2 (02), 147-167.

Schmidt, R. (1994). Implicit learning and the cognitive unconscious: Of artificial grammars and SLA. En N. Ellis (ed.). Implicit and explicit learning of languages (pp. 165-209). London: Academic Press.

Srichanyachon, N. (2012). Teacher written feedback for L2 learners' writing development. Journal of Social Sciences, Humanities, and Arts, 12 (1) 7-17.

Swain, M. (1995). Three functions of output in second language learning. Principle and practice in applied linguistics: Studies in honour of $\mathrm{HG}_{\mathrm{G}}$ Widdowson, 125-144.

Tatawy, M. (2002). Corrective feedback in second language acquisition. Working papers in Tesol \& Applied Linguistics, 2 (2), 1-19.

Truscott, J. (1996). The case against grammar correction in L2 writing classes. Language learning, 46 (2), 327-369.

VanPatten, B. (2011). Stubborn syntax: How it resists explicit teaching and learning. En C. Sanz \& R. Leow (Eds.), Implicit and explicit language learning: Conditions, processing, and knowledge (pp. 9-21). Washington, D. C.: Georgetown University Press.

Vygotsky, L. S. (1999). Tool and sign in the development of the child. The Collected works of L. S. Vygotsky, vol. 6. Scientific Legacy. Kluwer Academic/Plenum.

Yang, X. (2010). Modelling text as process: A dynamic approach to EFL classroom discourse. London: Continuum International Publishing Group.

Yule, G. (1996). Pragmatics. Oxford: Oxford University Press.

Zhang, S. (2012). Promoting noticing in EfL classroom. Theory and Practice in Language Studies, 2 (3), 579-584. 